\title{
Influence of functional gene polymorphisms on human behaviour: the case of CCR5
}

\author{
Paul R. Albert, PhD
}

The idea that genetic changes can impact behaviour and lead to novel therapeutic approaches for mental illness has been an attractive, yet elusive hypothesis. A couple of recent papers in JPN build the case for the effectiveness of maraviroc in ameliorating depression ${ }^{1}$ and reducing the addictive properties of opioids without preventing their analgesic effects. ${ }^{2}$ Maraviroc is an antagonist of C-C motif chemokine receptor-5 (CCR5) and has been shown prevent HIV infection by blocking its coreceptor CCR5. These new studies build on the finding that people with a 32-bp deletion mutation that inactivates CCR5 (CCR5- 132$)$ and show resistance to HIV also have improved cognitive outcomes after a stroke. ${ }^{3}$ The studies also extend a growing literature implicating CCR5 in mental illness and suggest that further clinical studies using maraviroc as a novel treatment or adjuvant, particularly in patients with inflammatory depression involving immune dysregulation, ${ }^{4}$ may be warranted. These findings suggest genetic polymorphisms with a strong functional impact (e.g., loss of function) may provide insight into mechanisms underlying certain forms of mental illness and lead to new treatments.

Much effort has been directed at studying genetic variants (such as polymorphisms) that can be used to identify risk of major depression or to improve its treatment. Improving diagnosis of depression may help overcome obstacles to treatment response. The first-line treatment for major depression remains the selective serotonin reuptake inhibitor (SSRI) class of antidepressants, ${ }^{5}$ even though these medications are effective in only $50 \%$ of patients, take several weeks to yield a response, and are associated with many adverse effects. ${ }^{6,7}$ Thus, alternative strategies, including augmentation or switching to alternative monoaminetargeting compounds, cognitive behavioural therapy (CBT) and various brain stimulation approaches, are indicated for patients with SSRI-resistant depression. Ultimately, about $30 \%$ of patients are treatment resistant, ${ }^{6}$ failing to respond to at least 3 treatments; most often electroconvulsive therapy or another form of brain stimulation is indicated, which may help half of these patients. ${ }^{8,9}$ Better alternatives are needed to treat major depression, and genetic approaches may reveal new drug targets that could be exploited for treatment-resistant depression.

\section{Genes and major depression}

Two main approaches have been used to identify genetic polymorphisms implicated in major depression or its treatment: candidate gene studies and genome-wide association studies (GWAS). ${ }^{10,11}$ Using animal and human models, candidate gene studies focus on polymorphisms in genes implicated in depression - ideally functional polymorphisms shown to affect gene function. Genome-wide studies provide an unbiased method of associating genetic change with phenotype. However, for complex diseases like major depression involving small contribution of many genes, hundreds of thousands of participants are required for sufficient statistical power. Identification of genetic polymorphisms associated with major depression or treatment response ${ }^{12}$ using genome-wide approaches has required enormous investment and resulted in few replicable polymorphisms across different studies and ethnicities, ${ }^{13}$ and each polymorphism contributes only a very small increase in risk for depression or treatment response. ${ }^{14-16}$ On the other hand, functional polymorphisms that have been shown to affect the function of a gene, either in vitro or in vivo, and to affect rodent behaviour (e.g., BDNF Val66Met rs6265), ${ }^{17}$ have not always been replicated in clinical association studies, nor in GWAS. ${ }^{10,11}$ Several factors could account for this variability, including the heterogeneity of the large GWAS sample sets, unreliability of diagnosis of depression, and changes in depression phenotype over time. ${ }^{18,19}$ There is a need to demonstrate functionality of human polymorphisms in human cells and ultimately in human behaviour. Polymorphisms that lead to genetic loss of function (e.g., copy number variations) are extremely rare, but some, like the CCR5- $\triangle 32$ polymorphism (allele frequency of 0.092 in Caucasian people), are abundant enough to be studied. This polymorphism was shown to confer resistance to HIV

Correspondence to: P. Albert, Ottawa Health Research Institute (Neuroscience), University of Ottawa, 451 Smyth Road, Ottawa, Ont K1H 8M5; palbert@uottawa.ca

Cite as: J Psychiatry Neurosci 2021 December 16;46(6). doi: 10.1503/jpn.210197 
infection since it disrupts the co-receptor that allows viral infection of the cell. ${ }^{20}$ More recently, this mutation has become highly contentious as an example of unethical germline gene manipulation in humans. In an infamous experiment to confer HIV resistance, the CCR5 mutation has been incorporated using CRISPR-Cas9 gene editing technology, resulting in 1 homozygous and 1 heterozygous mutation in a pair of twin girls. ${ }^{21}$ Nevertheless, the hereditary mutation and its effect on the brain has become of increasing interest with the finding that CCR5- $\Delta 32$ confers cognitive protection following stroke. ${ }^{3}$

\section{CCR5 and depression}

Recently, it was reported that in rodent models of stroke, Ccr5 expression is induced in cortical microglia and neurons within 12 hours post-stroke. Nine weeks of treatment with maraviroc (or gene knockdown of Ccr5 in the premotor cortex) was effective to promote recovery of motor function after photothrombotic focal stroke and of cognitive function after traumatic brain injury in C57BL6 mice. ${ }^{3}$ In the same study, human stroke patients from the TABASCO trial in Israel with the CCR5- $\triangle 32$ were found to have better scores on the NIH stroke scale (NIHSS). These patients also displayed greater scores on a battery of cognitive tests. These results suggest that genetic inactivation or pharmacological inhibition of CCR5 is associated with better sensorimotor and cognitive outcomes after stroke. In the July-August 2021 issue of JPN, Tene and colleagues examined depressive symptoms in the TABASCO cohort of stroke patients and showed that the CCR5- $\Delta 32$ patients had a significant reduction compared with noncarriers. ${ }^{1}$ While the severity of depression was mild in these patients, who had mild to moderate strokes, CCR5- $\Delta 32$ was associated with a significant reduction in depressive symptoms, which was most pronounced in participants having CCR5- $\Delta 32$ at both alleles. Interestingly, patients with the 5-HTTLPR-L allele and CCR5- $\Delta 32$ showed the greatest difference compared with noncarriers with the 5-HTTLPR-S allele, suggesting a potential interaction with the serotonin system. Consistent with this interaction, a recent observational study reported that antidepressant responders (SSRI/serotonin-norepinephrine reuptake inhibitors [SNRI]) showed reduced leukocyte RNA levels of CCR5 and its ligand CCL5 compared with patients who did not respond after 5 weeks of treatment. ${ }^{22}$ This effect could be driven by the action of SSRI treatment ex vivo to reduce macrophage CCR5 expression ${ }^{23}$ or by $5-\mathrm{HT}_{1 \mathrm{~A}}$ receptormediated downregulation of CCR5 in macrophages. ${ }^{24}$ Furthermore, compared with healthy controls, like other cytokines (e.g., interleukin-6), levels of CCR5 and CCL5 are increased in individuals with depression, the latter correlating with increasing severity. ${ }^{25}$ It remains to be seen whether patients with depression, particularly those with "inflammatory depression," in whom inflammatory cytokine and CCR5 levels are elevated, might benefit from treatment with maraviroc, or the combination of an SSRI and maraviroc.

\section{CCR5 and opioid addiction}

With regard to the role of CCR5 in modulating opioid actions, based on the heterodimerization and crossdesensitization of CCR5 and $\mu$-opioid receptors, ${ }^{26}$ Iriah and colleagues addressed the effect of maraviroc on oxycodone actions and addiction in rats, while monitoring changes in functional brain connectivity. ${ }^{2}$ Interestingly, maraviroc reduced conditioned place preference for oxycodone and oxycodone-induced functional connectivity of the reward circuitry. Maraviroc also selectively reduced oxycodone self-administration over 10 days compared with inactive lever and reduced drug-seeking after abstinence. However, maraviroc did not affect locomotion or oxycodone-induced analgesia in the tail flick test. These studies suggest that maraviroc preserves the antinociceptive actions of oxycodone while reducing its actions on brain circuitry associated with addiction. In mice, it was found that maraviroc or other CCR5 antagonists actually increased the potency of morphineinduced antinociceptive activity in 3 different tests. ${ }^{27}$ Similarly, maraviroc enhanced the potency of morphine to reduce incisional or cold-water pain in rats without affecting the dose for respiratory depression. ${ }^{28,29}$ Interestingly, a bivalent CCR5 antagonist- $\mu$-opioid agonist (MCC22) was several thousand times more potent an analgesic than the opioid agonist alone or combined with CCR5 antagonist, implicating receptor heterodimerization. ${ }^{30,31}$ Unlike with morphine, rats did not acquire tolerance or conditioned place preference to this bivalent ligand, suggesting that MCC22 may be useful as an analgesic in humans. ${ }^{32}$ It would be interesting to test whether opioid analgesia, tolerance or addiction has been reported in CCR5- $\Delta 32$ carriers.

But how is maraviroc-induced inhibition of morphine's addictive action induced? A clue comes from a study of cocaine-induced addiction in rats, which induces Ccr5 RNA expression in the mesolimbic reward centre. ${ }^{33}$ Treatment with maraviroc reduced conditioned place preference for cocaine and cocaine-induced locomotion, both actions mediated via the mesolimbic dopamine reward circuitry. This suggests that blockade of CCR5 may reduce dopamine activity, as seen in Ccr5-knockout mice ${ }^{34}$ thus reducing the addictive properties of both cocaine and opioids. It would be important to test whether maraviroc could also affect stress-induced drug seeking and reinstatement.

\section{Conclusion}

The evidence from preclinical pharmacological studies and clinical studies of carriers of the CCR5- $\Delta 32$ polymorphism, while still preliminary, favours a potentially important role of CCR5 inhibition in improving a variety of neuropsychiatric conditions, including major depression, opioid addiction, and stroke recovery. These studies highlight the importance of investigating established functional polymorphisms that have a large effect on gene expression 
(CCR5 loss of function) and demonstrated effects of human physiology (HIV resistance). We have previously emphasized the importance of considering the functional status of genetic polymorphisms, ${ }^{17}$ namely, a functional ranking of class 0 (no experimental evidence); 1 (in vitro evidence); 2 (cellular evidence) and 3 (in vivo evidence). Perhaps a class 4 (in vivo evidence in humans) is needed for polymorphisms that have clear evidence of a functional effect. Typically, the polymorphisms identified in GWAS remain completely in class 0 , with only bioinformatic predictions of what their function might be. ${ }^{14,35,36}$ Thus, little insight into specific mechanisms of disease have been provided to date. Perhaps using new leads like the CCR5- $\Delta 32$ polymorphism, it is time to refocus more on functional polymorphisms that affect behaviour to gain insights into disease mechanisms.

The case of the CCR5- $\triangle 32$ polymorphism illustrates how a single mutation in a single gene can impact human physiology, endowing resistance to HIV infection. Whether it also leads to benefits in mental and cognitive health remains to be further verified. Whole exome or genome sequencing may reveal rare polymorphisms with high functional impact, ${ }^{37}$ but thus far functional validation often remains unaddressed. Few mutations have been unequivocally linked to depression, but brain physiology can be highly impacted by single gene mutations. An example is narcolepsy induced by rare mutation of the hypocretin/orexin receptor gene, first found in dogs then in humans..$^{38}$ Subsequently, human narcolepsy was shown to involve loss of hypocretin neurons or reduced cerebrospinal fluid hypocretin levels in some, but not all, patients. This understanding has led to the development of orexin ligands to treat sleep disorders, including agonists for hypersomnia and antagonists for daytime sleepiness. ${ }^{39}$ Thus, understanding how specific genes contribute to brain function remains a largely under-explored and underfunded goal that needs greater focus if we are to understand what processes lead to alterations in human behaviour. As seen for the CCR5- $\Delta 32$ variant, it may be that gene variants associated with specific alterations in immune response could also impact behaviour and susceptibility to psychiatric disorders. On the other hand, patients with elevated levels of CCR5 or its ligand may be responsive to maraviroc. Identification of these functional variants and their mechanisms could inform treatment approaches to provide more targeted and effective personalized medicine. ${ }^{4,40}$

Affiliation: From the Ottawa Hospital Research Institute, University of Ottawa Brain and Mind Research Institute, Ottawa, Ont., Canada.

Competing interests: None declared.

Content licence: This is an Open Access article distributed in accordance with the terms of the Creative Commons Attribution (CC BY-NC-ND 4.0) licence, which permits use, distribution and reproduction in any medium, provided that the original publication is properly cited, the use is noncommercial (i.e., research or educational use), and no modifications or adaptations are made. See: https: / / creativecommons.org/licenses/by-nc-nd/4.0/

\section{References}

1. Tene $\mathrm{O}$, Hallevi $\mathrm{H}$, Molad J, et al. CCR5- $\Delta 32$ polymorphism: a possible protective factor for post-stroke depressive symptoms. $J$ Psychiatry Neurosci 2021;46:E431-40.

2. Iriah SC, Borges C, Shalev U, et al. The utility of maraviroc, an antiretroviral agent used to treat HIV, as treatment for opioid abuse? Data from MRI and behavioural testing in rats. J Psychiatry Neurosci 2021;46:E548-58.

3. Joy MT, Ben Assayag E, Shabashov-Stone D, et al. CCR5 is a therapeutic target for recovery after stroke and traumatic brain injury. Cell 2019;176:1143-57.e13.

4. Hayley S, Hakim AM, Albert PR. Depression, dementia and immune dysregulation. Brain 2021;144:746-60.

5. Kennedy SH, Lam RW, McIntyre RS, et al. Canadian Network for Mood and Anxiety Treatments (CANMAT) 2016 clinical guidelines for the management of adults with major depressive disorder: Section 3. Pharmacological treatments. Can J Psychiatry 2016;61:540-60.

6. Trivedi MH, Rush AJ, Wisniewski SR, et al. Evaluation of outcomes with citalopram for depression using measurementbased care in $\mathrm{STAR}^{*} \mathrm{D}$ : implications for clinical practice. $A m \mathrm{~J}$ Psychiatry 2006;163:28-40.

7. Cipriani A, Furukawa TA, Salanti G, et al. Comparative efficacy and acceptability of 21 antidepressant drugs for the acute treatment of adults with major depressive disorder: a systematic review and network meta-analysis. Lancet 2018;391:1357-66.

8. Milev RV, Giacobbe P, Kennedy SH, et al. Canadian Network for Mood and Anxiety Treatments (CANMAT) 2016 clinical guidelines for the management of adults with major depressive disorder: Section 4. Neurostimulation treatments. Can J Psychiatry 2016;61:561-75.

9. Kraus C, Kadriu B, Lanzenberger R, et al. Prognosis and improved outcomes in major depression: a review. Transl Psychiatry 2019;9:127.

10. Flint J, Kendler KS. The genetics of major depression. Neuron 2014;81:484-503.

11. Border R, Johnson EC, Evans LM, et al. No support for historical candidate gene or candidate gene-by-interaction hypotheses for major depression across multiple large samples. Am J Psychiatry 2019;176: 376-87.

12. Fabbri C, Kasper S, Kautzky A, et al. Genome-wide association study of treatment-resistance in depression and meta-analysis of three independent samples. Br J Psychiatry 2019;214:36-41.

13. Giannakopoulou O, Lin K, Meng X, et al. The genetic architecture of depression in individuals of East Asian ancestry: a genomewide association study. JAMA Psychiatry 2021;78:1258-69.

14. Wray NR, Ripke S, Mattheisen M, et al. Genome-wide association analyses identify 44 risk variants and refine the genetic architecture of major depression. Nat Genet 2018;50:668-81.

15. Howard DM, Adams MJ, Clarke TK, et al. Genome-wide metaanalysis of depression identifies 102 independent variants and highlights the importance of the prefrontal brain regions. Nat Neurosci 2019;22:343-52.

16. Amare AT, Schubert KO, Hou L, et al. Association of polygenic score for major depression with response to lithium in patients with bipolar disorder. Mol Psychiatry 2021;26:2457-70.

17. Albert PR. What is a functional genetic polymorphism? Defining classes of functionality. J Psychiatry Neurosci 2011;36:363-5.

18. Alda M. Psychiatric genetics - Does diagnosis matter? J Psychiatry Neurosci 2017;42:291-3.

19. Alda M. The moving target of psychiatric diagnosis. J Psychiatry Neurosci 2021;46:E415-17.

20. Samson M, Libert F, Doranz BJ, et al. Resistance to HIV-1 infection in Caucasian individuals bearing mutant alleles of the CCR-5 chemokine receptor gene. Nature 1996;382:722-5.

21. Cyranoski D, Ledford H. Genome-edited baby claim provokes international outcry. Nature 2018;563:607-8.

22. Bauer O, Milenkovic VM, Hilbert S, et al. Association of chemokine (C-C Motif) receptor 5 and ligand 5 with recovery from major depressive disorder and related neurocognitive impairment. Neuroimmunomodulation 2020;27:152-62. 
23. Greeson JM, Gettes DR, Spitsin S, et al. The selective serotonin reuptake inhibitor citalopram decreases human immunodeficiency virus receptor and coreceptor expression in immune cells. Biol Psychiatry 2016;80:33-9.

24. Manéglier B, Guillemin GJ, Clayette P, et al. Serotonin decreases HIV-1 replication in primary cultures of human macrophages through 5-HT(1A) receptors. Br J Pharmacol 2008;154:174-82.

25. Ogłodek EA, Szota A, Just MJ, et al. Comparison of chemokines (CCL-5 and SDF-1), chemokine receptors (CCR-5 and CXCR-4) and IL-6 levels in patients with different severities of depression. Pharmacol Rep 2014;66:920-6.

26. Chen C, Li J, Bot G, et al. Heterodimerization and crossdesensitization between the mu-opioid receptor and the chemokine CCR5 receptor. Eur J Pharmacol 2004;483:175-86.

27. Eisenstein TK, Chen $X$, Inan $S$, et al. Chemokine receptor antagonists in combination with morphine as a novel strategy for opioid dose reduction in pain management. Mil Med 2020;185:130-5.

28. Inan $\mathrm{S}$, Eisenstein $\mathrm{TK}$, Watson $\mathrm{MN}$, et al. Coadministration of chemokine receptor antagonists with morphine potentiates morphine's analgesic effect on incisional pain in rats. J Pharmacol Exp Ther 2018;367:433-41.

29. Inan S, Chen X, Eisenstein EM, et al. Chemokine receptor antagonists enhance morphine's antinociceptive effect but not respiratory depression. Life Sci 2021;285:120014.

30. Akgün E, Javed MI, Lunzer MM, et al. Inhibition of inflammatory and neuropathic pain by targeting a mu opioid receptor/ chemokine receptor5 heteromer (MOR-CCR5). J Med Chem 2015;58:8647-57.

31. Portoghese PS, Akgün E, Lunzer MM. Heteromer induction: an approach to unique pharmacology? ACS Chem Neurosci 2017;8:426-8.
32. Cataldo G, Erb SJ, Lunzer MM, et al. The bivalent ligand MCC22 potently attenuates hyperalgesia in a mouse model of cisplatin-evoked neuropathic pain without tolerance or reward. Neuropharmacology 2019;158:107598.

33. Nayak SU, Cicalese S, Tallarida C, et al. Chemokine CCR5 and cocaine interactions in the brain: Cocaine enhances mesolimbic CCR5 mRNA levels and produces place preference and locomotor activation that are reduced by a CCR5 antagonist. Brain Behav Immun 2020;83:288-92.

34. Choi DY, Lee MK, Hong JT. Lack of CCR5 modifies glial phenotypes and population of the nigral dopaminergic neurons, but not MPTP-induced dopaminergic neurodegeneration. Neurobiol Dis 2013;49:159-68.

35. Network and Pathway Analysis Subgroup of Psychiatric Genomics Consortium. Psychiatric genome-wide association study analyses implicate neuronal, immune and histone pathways. Nat Neurosci 2015;18:199-209.

36. Wray NR, Pergadia ML, Blackwood DH, et al. Genome-wide association study of major depressive disorder: new results, metaanalysis, and lessons learned. Mol Psychiatry 2012;17:36-48.

37. Yu C, Arcos-Burgos M, Baune BT, et al. Low-frequency and rare variants may contribute to elucidate the genetics of major depressive disorder. Transl Psychiatry 2018;8:70.

38. Mignot E. Sleep, sleep disorders and hypocretin (orexin). Sleep Med 2004;5(Suppl 1):S2-8

39. Sun Y, Tisdale RK, Kilduff TS. Hypocretin/orexin receptor pharmacology and sleep phases. Front Neurol Neurosci 2021;45:22-37.

40. Milaneschi Y, Lamers F, Berk M, et al. Depression heterogeneity and its biological underpinnings: toward immunometabolic depression. Biol Psychiatry 2020;88:369-80. 